\title{
Modelo computacional para visualizar desplazamientos atómicos en cascada de materiales sometidos a radiación ionizante
}

\section{Computer model to visualize atomic cascade displacements of materials subjected to ionizing radiation}

págs. 71-81
Grupo de Investigación: Diseño avanzado
Línea de investigación: modelado computacional
Jhon Freddy Ochoa-Avendaño•, Miguel Alfonso Morales Granados*.

\begin{abstract}
Resumen
Este artículo presenta un modelo numérico simplificado para visualizar el proceso de desplazamientos en cascada de los átomos de un material. Esto se logra a través de una implementación computacional del potencial de Lennard-Jones y de la solución de las ecuaciones de movimiento mediante el algoritmo de Verlet. Como resultado del código desarrollado, es posible determinar la cantidad de átomos que se desplazan de sus posiciones iniciales.
\end{abstract}

Palabras clave: Daños por radiación, Potencial de Lennard-Jones, Dinámica Molecular, Algoritmo de Verlet.

\begin{abstract}
The purpose of this article is present a simplified model to visualize the displacement cascade process in the atoms of a material. This achievement can be accomplished through a computational modeling method, this method will use a computational implementation of a Lennard-Jones function and the motion equations solution through a Verlet Algorithm. As a result of the developed code, the amount of atoms displaced can be calculated from its initial position.
\end{abstract}

Keywords: Radiation Damage, Lennard-Jones Potential Function, Molecular Dynamics, Verlet Integration Algorithm.

- Ingeniero Mecánico, Docente investigador. Fundación Universidad de América. jhon.ochoa@profesores.uamerica.edu.co

-• Ingeniero Mecánico, docente investigador. Fundación Universidad de América. miguel.morales@investigadores.uamerica.edu.co 


\section{Introducción}

La interacción de radiaciones ionizantes con los metales, produce la degradación progresiva en estos materiales. El daño se producen cuando las partículas que viajan grandes velocidades ${ }^{1}$ ingresan al material produciendo cambios en los átomos de su estructura cristalina(Schilling \& Ullmaier, 2006). Las alteraciones a nivel cristalográfico dependen del tipo de interacción que ocurra entre la partícula proyectil y los átomos del material; a su vez, dichas alteraciones producen diferentes tipos de daño(Guernet, Bruel, Gailliard, Garcia, \& Robic, 1977).

Existen dos tipos de interacciones: La primera recibe el nombre de Interacción inelástica. En este tipo de interacción la partícula proyectil viaja con la suficiente energía como para penetrar el núcleo de uno de los átomos del material. Esto puede producir una reacción físico-química, dando lugar a un proceso de fisión o cambios químicos en el material (Davies \& Ewart, 1971).

El segundo tipo de interacción recibe el nombre de Interacción elástica. En este caso la partícula proyectil viaja con una energía menor a la necesaria para penetrar el núcleo de alguno de átomos del material, por lo que ocurre un choque elástico que podría llegar a desplazar de su posición inicial al átomo que recibe el impacto ${ }^{2}$ (Hudson, Dudarev, Caturla, \& Sutton, 2005).

Los dos tipos de interacción producen cambios en la estructura cristalina del material, modificando sus propiedades físicas y su comportamiento mecánico. Sin embargo los desplazamientos de átomos de sus posiciones de red cristalina, son el inicio de la mayor cantidad de daños progresivos en los metales. Tanto así, que para el diseño de reactores nucleares, son mayormente consideradas las interacciones elásticas entre neutrones rápidos y átomos del

1 Para efectos de éste documento la partícula en adelante se denominará partícula proyectil.

2 En adelante se le denominará blanco al átomo que recibe el primer impacto elástico. material irradiado (Manrique, 2005). El presente artículo se basa en las interacciones de este tipo y toma como ejemplo de explicación las condiciones de operación de las paredes de un reactor nuclear, donde el material está sometido a una alta radiación neutrónica. En la figura 1 se presenta un esquema de la interacción elástica (Clément, 2010).

Figura 1. Interacción elástica entre un neutrón y uno de los átomos del material irradiado.

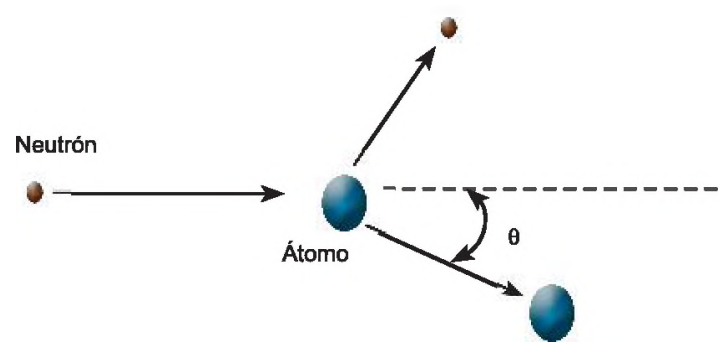

Fuente: (Clément, 2010).

\section{Materiales y métodos}

\subsection{Definiciones teóricas iniciales}

Daños por radiación en materiales

En los reactores nucleares la probabilidad de colisión entre neutrones rápidos y un átomo del material es calculada por medio de la ecuación 1, (Clément, 2010). Se define como el número de interacciones elásticas e inelásticas por unidad de volumen, de tal manera que:

$$
N_{i}=N_{v} \sigma(E) \varphi_{n}(E)
$$

$N_{v}$ es el número de átomos que podrían interactuar en la unidad de volumen, $\phi n(E)$ es el flujo de neutrones en el rango de energía considerado y $\sigma(E) \sigma(E)$ es conocido cómo interacción en la sección transversal ${ }^{3}$, parámetro que describe la probabilidad de cada tipo de interacción (Koutský \& Kočík, 1994).

3 Traducción del concepto Interaction Cross Section 
LÍNEA DE INVESTIGACIÓN: MODELADO COMPUTACIONAL

Al considerar solamente las interacciones elásticas, se parte del hecho de que no ocurre una modificación en la estructura de los átomos del material, pero parte de la energía cinética del neutrón se transfiere al átomo impactado (Clément, 2010). Esto se puede describir por medio de la ecuación 2.

$$
E_{i}=4 E_{n} \cos ^{2}(\theta) \frac{m_{n} m_{t}}{\left(m_{n}+m_{T}\right)^{2}}
$$

$E_{T}$ corresponde a la energía cinética transferida al blanco, $m_{n}$ y $m_{T}$ son las masa del neutrón y el blanco respectivamente; $E_{n}$ es la energía del neutrón antes de la colisión y $\theta$ es el ángulo de incidencia del neutrón, ver figura 1 . Por otra parte, se define $\lambda$ como parámetro de energía transferida de la siguiente manera (Osetsky, Bacon, Singh, \& Wirth, 2002):

$$
\lambda=\frac{E_{I}}{E_{N}}
$$

Dependiendo del valor de $\lambda$, la energía transferida genera diferentes alteraciones al material irradiado; para valores pequeños, el átomo impactado permanece en su sitio dentro del arreglo cristalino. Para valores de energía transferida entre 20-40 eV, el blanco es desplazado de su posición intersticial en la red cristalina. El valor crítico de energía para que un átomo sea retirado de su posición recibe el nombre de Energía de Desplazamiento $E_{d^{2}}$ valor que puede ser tomado de la norma ASTM E-521-96 para distintas aleaciones (Clément, 2010).

\section{Desplazamientos en cascada}

Si después de la colisión el blanco adquiere una alta cantidad de energía, este podría desplazarse con velocidad considerable y chocar contra los demás átomos de la red cristalina produciendo movimiento en estos últimos. Este proceso recibe el nombre de Desplazamiento en Cascada, es uno de los daños más recurrente en materiales sometidos a radiación (Manrique, 2005). En este caso, el primer átomo colisionado por el neutrón recibe el nombre de
PKA4. Norgett y colaboradores (Norgett, Robinson, \& Torrens, 1975) presentan la ecuación 4 para calcular el número de átomos desplazados en cascada.

$$
N_{N R T}=0.8 \frac{E_{p k a}}{E_{d}}
$$

Asumiendo que el tipo de interacción entre el neutrón y el PKA es igual al tipo de interacción entre el PKA y el siguiente átomo chocado, se podría calcular la energía transferida desde el PKAal siguiente átomo por medio de la ecuación.

Por otra parte, si la cantidad de átomos impactados por neutrones que se convierten PKA se multiplica por el valor la cantidad de átomos desplazados en cascada, se obtiene como resultado una magnitud del daño por radiación en términos de desplazamientos atómicos en el material, $D P A_{N R T}$ Esto se puede cuantificar acorde con la ecuación 5 .

$$
D P A_{N R T}=\frac{\rho N}{M_{a}} \sigma \emptyset \frac{E_{n}}{E_{d}} \frac{1.6\left(m_{T}+m_{n}\right)}{\left(m_{T} m_{n}\right)^{2}}
$$

En la anterior expresión pes la masa específica del material, $N$ corresponde al número de Avogadro, $M_{a}$ es la masa atómica del material, $\sigma$ es la interacción en la sección transversal para la energía considerada, $\phi$ es el flujo de neutrones y $E_{n}$ la energía cinética del neutrón incidente. Se debe mencionar que la ecuación 5 , no tiene en cuenta todo el rango de energia que pueden tener los neutrones incidentes. Para dar solución a lo anterior, Clément (Clément, 2010) plantea la razón de formación de desplazamientos de la siguiente manera:

$$
R_{d}=N \int_{\frac{E_{d}}{\lambda}}^{\infty} \varphi\left(E_{n}\right) d E_{n}+\int_{E_{d}}^{\lambda E_{n}} \sigma_{n}\left(E_{n}, T\right) \nu(T) d T
$$

donde $N$ es el número de átomos por unidad de volumen, $\lambda$ es el parámetro de energía transferida definido anteriormente en la ecuación 3.

4 Acrónimo de Primary Knocked Atom 
La segunda integral de la anterior expresión, recibe el nombre de desplazamiento en la sección transversal y se denota con $\sigma d\left(E_{n}\right)$. Dicho valor se encuentra tabulado para distintos materiales en (Clément, 2010).

$$
\sigma_{d}\left(E_{n}\right)=\int_{E_{d}}^{\lambda E_{n}} \sigma_{n}\left(E_{n}, T\right) \nu(T) d T
$$

\section{Dinámica molecular}

Los modelos basados en dinámica molecular permiten realizar cálculos de las trayectorias de los átomos contenidos en un volumen de control. Para esto, se considera la interacción entre átomos de acuerdo con el potencial interatómico entre ellos.

Estos modelos han sido usados desde los años 60 para explicar la evolución del daño de materiales irradiados, dada la aparición de desplazamientos atómicos en cascada. También, permiten la implementación de simulaciones con millones de átomos, resultando bastante útil para calcular el tiempo de evolución de desplazamientos en cascada en un caso real. Adicionalmente es posible obtener estimativos sobre el desarrollo de dislocaciones en los cristales de material, así como cálculos de los esfuerzos debido a las temperaturas (Osetsky et al., 2002)

A continuación se presenta la ecuación diferencial general de la dinámica molecular, que describe el movimiento de los átomos dentro de la red cristalina. En esta se relaciona la fuerza de cada partícula, con su energía cinética.

$$
m_{i} \frac{\partial r_{i, j}}{\partial t^{2}}=\frac{\partial r_{i, j}}{\partial r_{i}} E_{T} ; i=1,2, \ldots, N
$$

\subsection{Modelo Computacional}

Potencial de Lennard-Jones

La función de potencial de Lennard-Jones, es una de las formas más usadas para describir la interacción de dos átomos en la forma de potencial (Jónsson \& Alexandersson, 2005). Esta se presenta en la ecuación 9.

$$
v(r)=4 \varepsilon\left(\left(\frac{\sigma}{r}\right)^{12}-\left(\frac{\sigma}{r}\right)^{6}\right)
$$

El primer término dentro del paréntesis de la ecuación 9 corresponde al potencial de repulsión y el segundo al potencial de atracción, $r$ es la distancia entre dos átomos, $\varepsilon$ es llamado la profundidad del potencial, ver figura 2 y $\sigma$ es la distancia mínima entre los átomos; corresponde a la distancia a la que los átomos empiezan a repelerse.

Figura 2.

Gráfica del Potencial de Lennard-Jones.

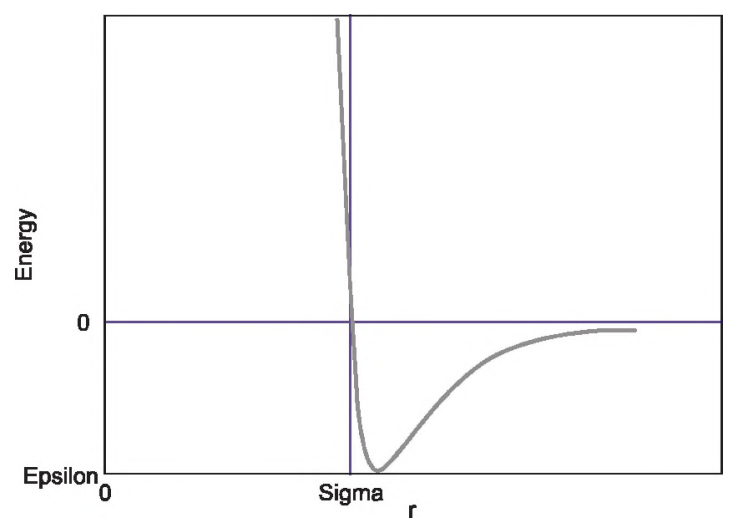

Fuente:(Elenius \& Oppelstrup, 2006).

Dado el potencial y la separación existente entre un par de átomos, se puede calcular la fuerza de atracción o repulsión entre ellos. Esto se obtiene al derivar la ecuación 10 con respecto $r$.

$$
\vec{F}=-\frac{\nabla v}{r}=\frac{24 \varepsilon}{r}\left(\left(\frac{\sigma}{r}\right)^{12}-\left(\frac{\sigma}{r}\right)^{6}\right)
$$


LÍNEA DE INVESTIGACIÓN: MODELADO COMPUTACIONAL

En la figura 3, se muestran las gráficas para el potencial de Lennard-Jones y su derivada para un par de partículas.

Figura 3.

Gráfica de potencial y fuerza de Lennard-Jones, fuente: (Manrique, 2005)

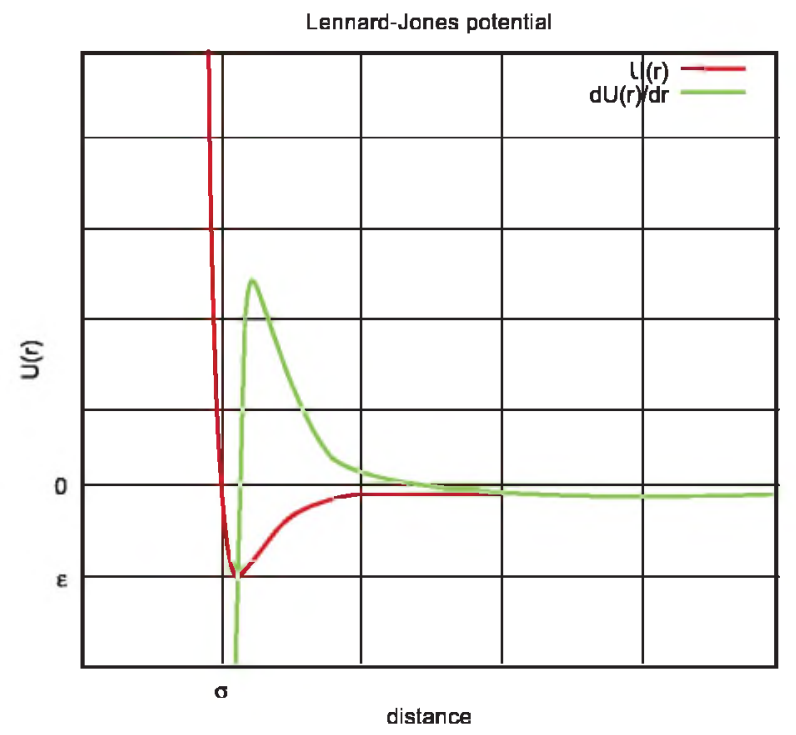

De manera que a partir de la 10 y con los valores de la masa se puede calcular la aceleración de los átomos. Esto es de gran utilidad para describir las trayectorias del movimiento de los mismos.

Integración de las ecuaciones de movimiento

Los valores de las aceleraciones de los átomos para cada instante de tiempo, permiten hacer el cálculo de la velocidad y posición de los átomos durante su interacción. En el presente artículo, se hace por medio del algoritmo de Verlet, expresado con las ecuaciones 11 y 12 .

$$
\begin{gathered}
r(t+\Delta t)=r(t)+v(t) \Delta t+\frac{1}{2} a(t) \Delta t^{2} \\
v(t+\Delta t)=v(t) \Delta t+\frac{1}{2} a(t) \Delta t
\end{gathered}
$$

\subsection{Implementación Computacional}

A continuación se describe la implementación computacional realizada para el desarrollo de este artículo.

\section{Problema desarrollado}

En el presente trabajo se implementó un algoritmo para simular numéricamente el proceso de desplazamientos en cascada de una serie de átomos ubicados dentro de un cubo. Se simularon las trayectorias de movimiento de átomos que son chocados por una partícula que proviene del exterior del cubo. En un caso ideal, la partícula del exterior debería ser un neutrón. Sin embargo, en el presente modelo, la partícula exterior corresponde a un átomo de igual masa $m=1$ que los átomos que se encuentran dentro del cubo. En la figura 4, se muestran las posiciones iniciales de los átomos. Se muestra que estos están organizados de forma equidistante dentro de cubo, adicionalmente, el átomo exterior, al que se le da el nombre de átomo proyectil es igualmente mostrado.

En la implementación computacional de las velocidades iniciales de los átomos, se consideraron valores bajos para los átomos dentro de cubo y un valor alto para el átomo proyectil. Sin embargo, la velocidad de éste último solo se da en una componente (en este caso $x$ ), ya que de ésta forma se garantiza una velocidad rectilínea uniforme hasta antes de la colisión inicial.

Como se habló en la sección 1.1. Dinámica Molecular, es posible simular el movimiento de los átomos por medio de la solución de la ecuación 8.

En el caso de este artículo, la interacción se realizó entre pares de átomos por medio de la función de potencial de Lennard-Jones presentada en la ecuación 9 , de manera que cada átomo interactúa con todos los demás. El cálculo de cada potencial se determina sumando el potencial anterior, es decir, el potencial de cada átomo es la suma de los potenciales que resultan su interacción con cada uno de los demás átomos, ecuación 13. 
Figura 4.

Posiciones iniciales de los átomos antes de la colisión.

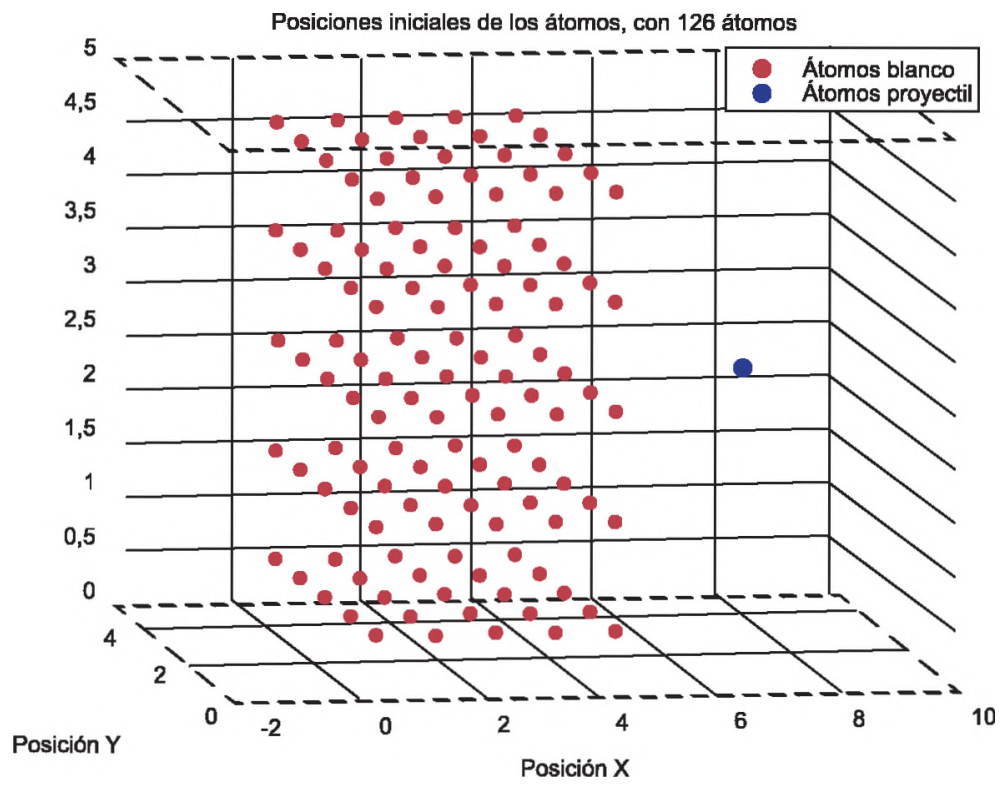

Fuente: autores.

\section{Cálculo del potencial}

Como se habló en la sección 1.1-Dinámica Molecular, es posible simular el movimiento de los átomos por medio de la solución de la ecuación 8.

En el caso de este artículo, la interacción se realizó entre pares de átomos por medio de la función de potencial de Lennard-Jones presentada en la ecuación 9, de manera que cada átomo interactúa con todos los demás. El cálculo de cada potencial se determina sumando el potencial anterior, es decir, el potencial de cada átomo es la suma de los potenciales que resultan su interacción con cada uno de los demás átomos, ecuación 13.

$$
v_{N}(r)=\sum_{i=1}^{N-1} v(r)
$$

La fuerza de interacción es calculada independientemente para las direcciones $X, Y$ y $Z$ y .
Descripción del movimiento

El movimiento de los átomos se determinó por medio del algoritmo de Verlet presentado en las ecuaciones 11 y 12. La variación del tiempo $\Delta t$, se estableció como un valor muy pequeño (del orden de $0.000001 \mathrm{~s}$ ). Pues como se muestra en la figura 2, para valores muy pequeños de el potencial tiende a infinito, lo que a su vez lleva a una fuerza y una aceleración muy grandes. El hecho de hacer los cálculos de posición y velocidad con aceleraciones muy altas, hace que los valores de desplazamiento sean igualmente altos. Esto limita la visualización de las trayectorias descritas por los átomos en el computador. Podría lograrse una mejor visualización de los resultados al utilizar valores de aún de menor orden, sin embargo el costo computacional sería muy alto, requiriendo un equipo de mayor capacidad.

\section{Condiciones de frontera}

Se situaron átomos dentro de un cubo, ver figura 4 con el objetivo de imitar las posiciones 
LÍNEA DE INVESTIGACIÓN: MODELADO COMPUTACIONAL

de los átomos en las celdas cristalinas de un metal. El cubo hace la vez de varias celdas cristalinas unidas formando un solo material. Los átomos son obligados a permanecer dentro del cubo a pesar de sus desplazamientos, pues rara que un átomo pueda escapar del material al que pertenece, debe adquirir una energía superior a la barrera de potencial existente en la superficie del material (Kishine \& Ovchinnikov, 2015).

Los cálculos realizados generalmente hacen que las posiciones de los átomos sean coordenadas por fuera del cubo para el instante $(t+\Delta t)$.
En el algoritmo acá implementado, fue necesario aplicar una subrutina que se encargara de identificar los átomos que eventualmente saldrían del cubo y de devolverlos cerca de la pared del mismo. Una vez que los átomos ingresaban al cubo nuevamente, éstos adquirían un movimiento contrario al inicial, es decir, se asume que los átomos llegan a la frontera del cubo y colisionan con ésta de forma perfectamente elástica.

Procedimiento de la implementación.

A continuación, en la figura 5 se presenta el diagrama de flujo del algoritmo implementado para realizar los cálculos.

Figura 5.

Diagrama de flujo del algoritmo principal implementado.

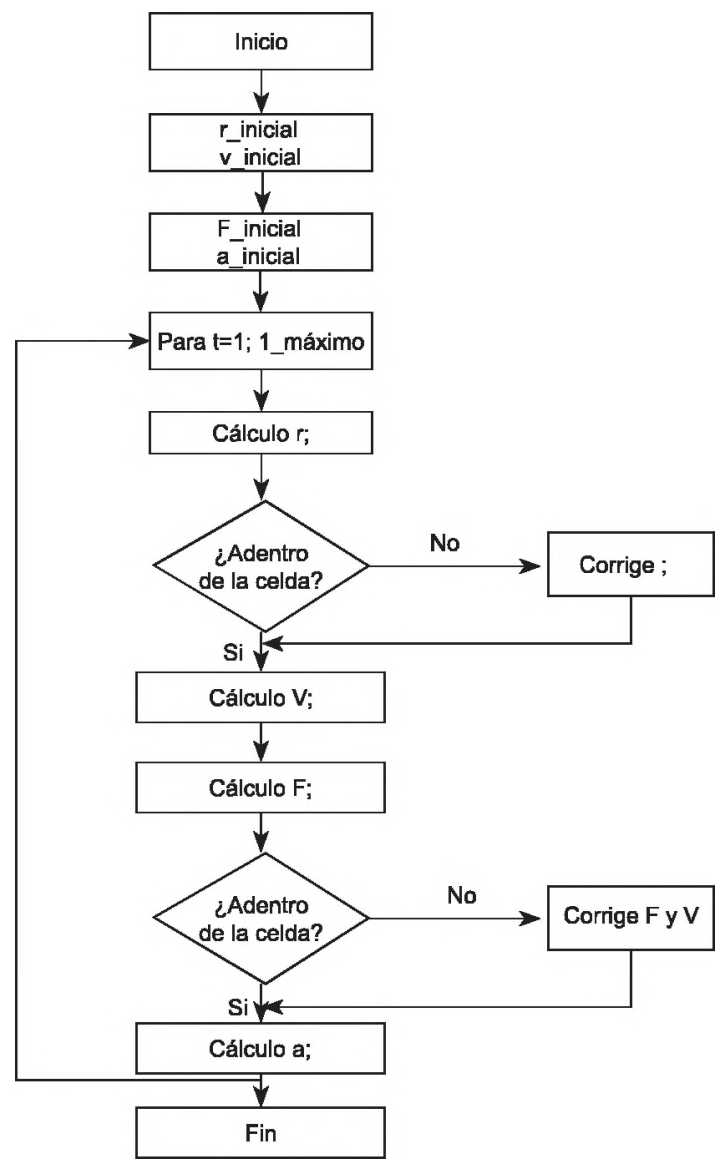

Fuente: autores 


\section{Materiales y Métodos}

Inicialmente se obtuvieron las gráficas de las figuras 6 y 7 donde se presentan el potencial y la fuerza tanto del modelo implementado como el valor teórico para un par de partículas.
Estos resultados se obtuvieron para realizar una verificación de las funciones implementadas. Una vez se obtuvieron los resultados esperados para un par de partículas, se evaluó la misma implementación con un mayor número de átomos.

Figura 6.

Comparación entre potencial implementado y potencial teórico de Lennard-Jones para un par de átomos.

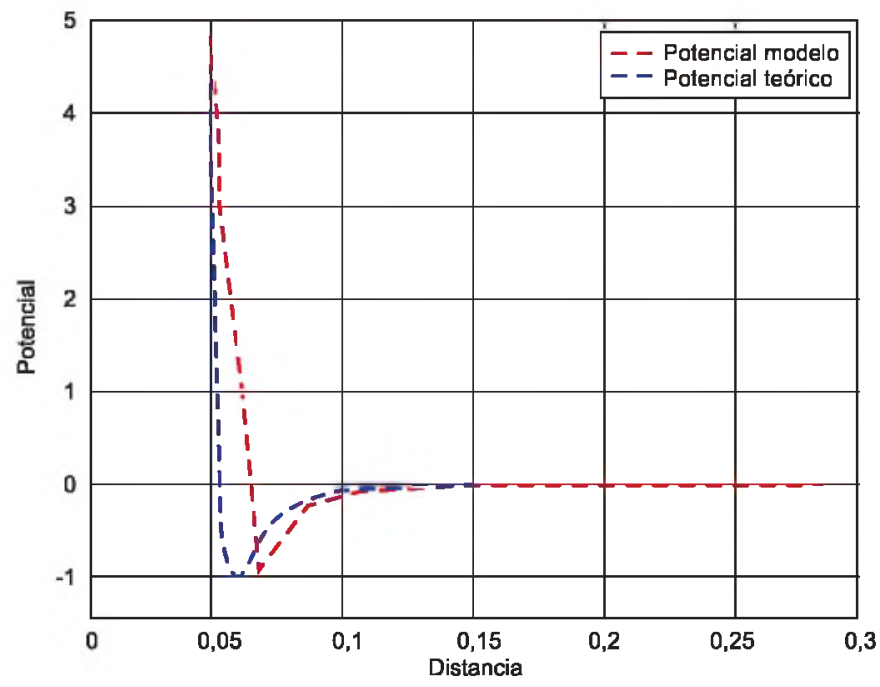

Fuente: Autores

Figura 7.

Comparación entre la fuerza del algoritmo implementado y la fuerza teórica para un par de átomos.

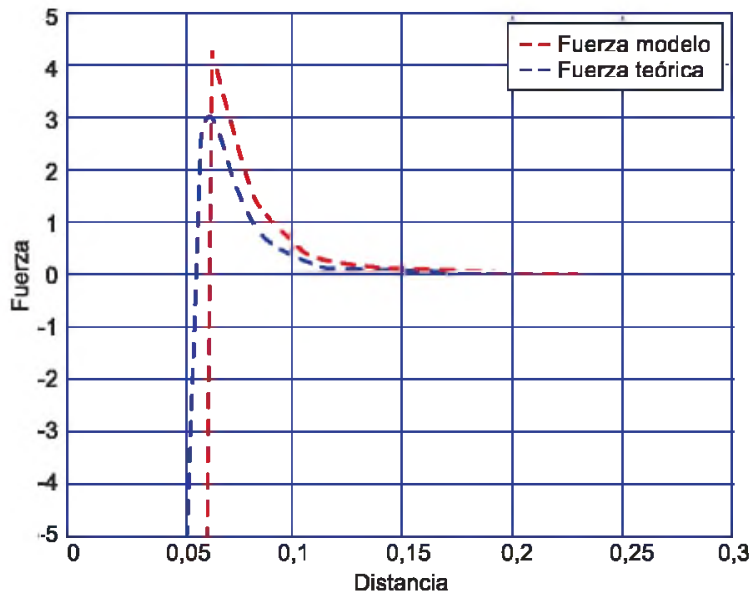

Fuente: autores. 
LÍNEA DE INVESTIGACIÓN: MODELADO COMPUTACIONAL

Para la visualización de los resultados, se generó un video del movimiento de los átomos dentro del cubo. Sin embargo para poder presentar resultados en el presente documento, se muestran las figuras 8 y 9 . En la primera, se observa en color rojo, los átomos que no fueron movidos de sus posiciones iniciales y en color azul, los átomos que fueron desplazados por las colisiones en cascada.

\section{Figura 8.}

Representación de los átomos que fueron desplazados por las colisiones en cascada.

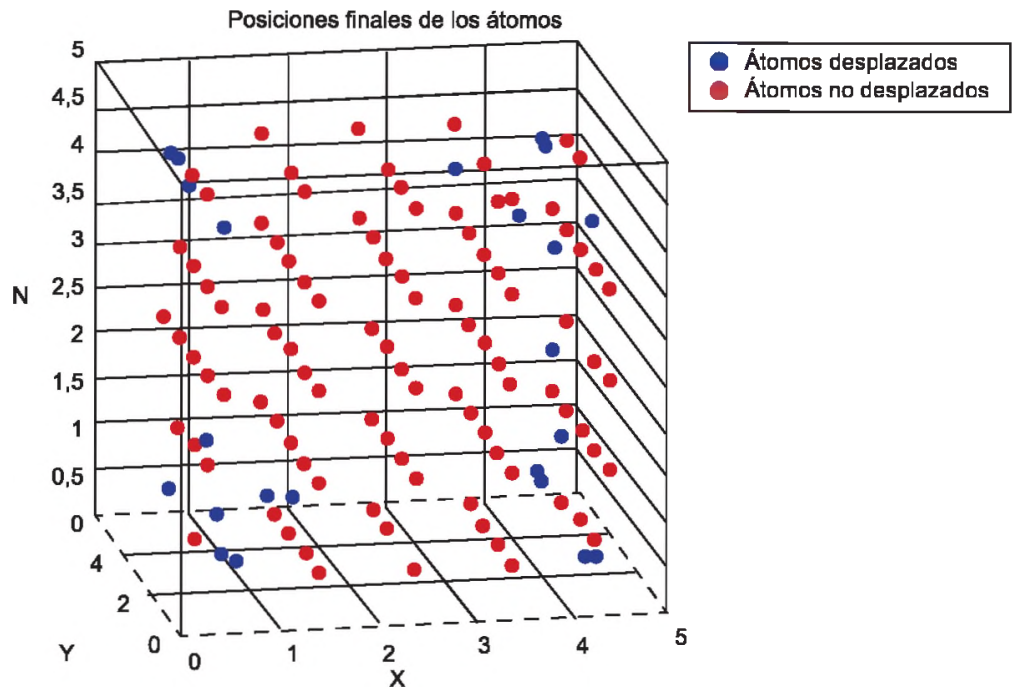

Fuente: autores.

Por su parte en la figura 9 , se presenta en forma cuadrados azules los sitios que originalmente fueron ocupados por átomos y que pos-

terior a la simulación aparecen con ausencia de átomos, esto representa las vacancias dentro del material.

\section{Figura 9.}

Representación de las vacancias que se presentan en el material al final de las colisiones en cascada.

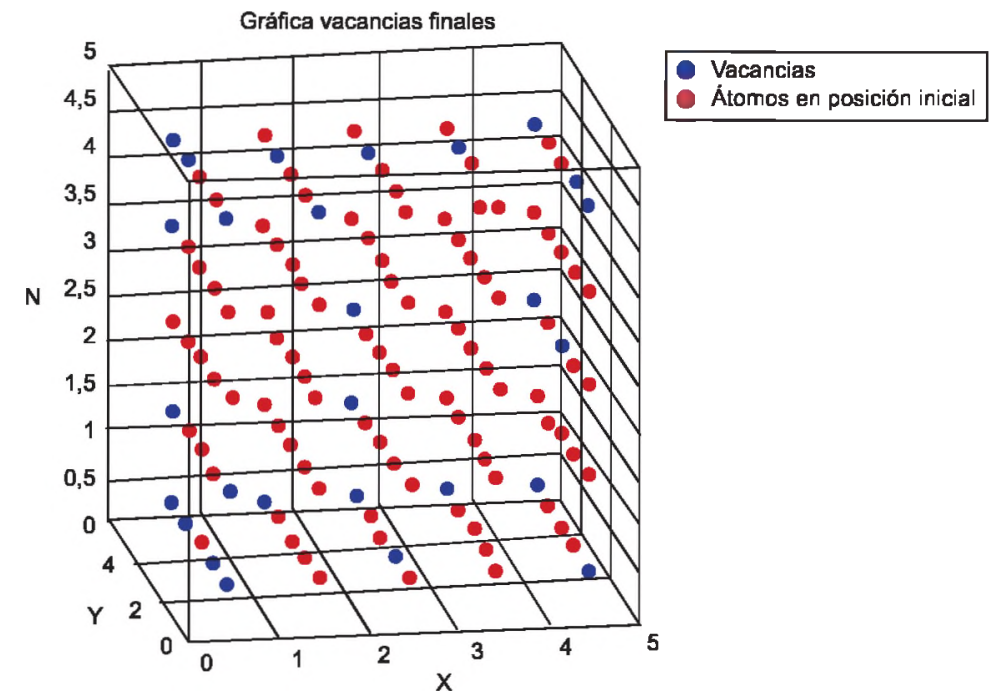

Fuente: autores 


\section{Comentarios}

En las figuras 6 y 7, se puede observar la similitud entre las curvas teóricas y las obtenidas mediante el algoritmo acá implementado. Allí es posible notar algunas diferencias, ya que en el modelo no se obtiene los puntos suficientes como para que las gráficas se sobrepongan. La carencia de puntos se debe a que la distancia entre un par de átomos, es calculada con base a la aceleración y como se mencionó anteriormente, para distancias muy pequeñas, los valores de potencial, fuerza y aceleración se vuelven demasiado grandes. Esto impide la aparición de valores cercanos a $r=0$, punto donde más difieren las gráficas. La forma de controlar los valores de $r, v, F$ y se logra introduciendo valores muy pequeños de $\mathrm{D} t$ en el algoritmo presentado. Sin embargo, aunque en el presente modelo se asignó un valor pequeño, es necesario que $\Delta t$ sea del orden de picosegundos.

En las gráficas 8 y 9 se observa como los átomos son desplazados de sus posiciones iniciales generando vacancias dentro del material. Esto implica la formación de una serie de defectos cristalinos que a su vez generan cambios en las propiedades mecánicas y en el comportamiento mecánico del material (Armstrong \& Britton, 2014). Al aplicar cargas a un sólido con vacancias, pueden producirse dislocaciones, las cuales empiezan a desplazarse por dentro del material como resultado de las deformaciones a las que el sólido es sometido. Cuando las dislocaciones llegan a la superficie se convierten en microgrietas; estas dan origen a proble- mas de fatiga en el material, razón por la que se estudian los desplazamientos en cascada.

En términos generales los resultados presentados corresponden a lo esperado, pues como se mencionó en la sección introductoria, las interacciones elásticas producen desplazamientos atómicos en cascada en el modelo. El trabajo desarrollado y presentado en éste artículo pretende ilustrar el comportamiento de los átomos durante este tipo de procesos. Sin embargo, es importante aclarar que los resultados acá obtenidos están lejos de ser un modelo aplicado a casos reales, pues se han hecho bastantes simplificaciones y suposiciones. Una de ellas es el hecho de que no sea un neutrón el que empieza las colisiones contra los átomos, sino un mismo átomo. Principalmente existe una diferencia sustancial entre las masas del neutrón y los átomos. Por lo tanto para obtener valores comparables con la realidad, haría falta aplicar una serie de procedimientos que pueden ocurrir en un proceso real. Los modelos aplicables poseen características tanto de colisiones elásticas como inelásticas y tienen en cuenta las cargas eléctricas producto de la descompensación que ocurre al desplazar un átomo de su posición original. Dichos modelos contemplan la posibilidad de que un átomo que sea desplazando de su posición original, quede atrapado en una vacancia existente. Otra característica de los modelos avanzados, es la posibilidad de formación de moléculas entre los átomos desplazados y no desplazados. Esto último podría ocurrir especialmente en materiales aleados, lo que implica una gran cantidad de compuestos en su composición química.

\section{Referencias}

Armstrong, D. E. J., \& Britton, T. B. (2014). Effect of dislocation density on improved radiation hardening resistance of nano-structured tungsten-rhenium. Materials Science and Engineering: A, 611, 388-393.

Clément, L. (2010). Nuclear materials and irradiation effects. Handbook of nuclear engineering (Cacuci,Dan.Gabriel. ed., pp. 543-642). Karlsruhe, Germany: Springer US. doi:978-0-38798149-9 
LÍNEA DE INVESTIGACIÓN: MODELADO COMPUTACIONAL

Davies, J. H., \& Ewart, F. T. (1971). The chemical effects of composition changes in irradiated oxide fuel materials. Journal of Nuclear Materials, 41(2), 143-155.

Elenius, M., \& Oppelstrup, T. (2006). In School of Computer Science and Communication (Ed.), Molecular dynamics simulations, lab 2. http://www.nada.kth.se/ tomaso/miklab2007/ lab2_4h1725.pdf: KTH Royal Institute of Technology.

Guernet, G., Bruel, M., Gailliard, J. P., Garcia, M., \& Robic, J. Y. (1977). Ion implantation in semiconductors and other materials.

Hudson, T. S., Dudarev, S. L., Caturla, M. J., \& Sutton, A. P. (2005). Effects of elastic interactions on post-cascade radiation damage evolution in kinetic monte carlo simulations. Philosophical Magazine, 85(4-7), 661-675.

Jónsson, H., \& Alexandersson, K. (2005). PhD thesis - classical trajectories (University of Iceland).

Kishine, J., \& Ovchinnikov, A. S. (2015). Chapter one - theory of monoaxial chiral helimagnet. Solid State Physics, 66, 1-130.

Koutský, J., \& Kočík, J. (1994). In Elsevier (Ed.), Radiation damage of structural materials (Materials science monographs 79 ed.). Amsterdam: Elsevier.

Manrique, C. B. (2005). PhD tesis - estudio del daño por radiación en aceros ferríticos (Universitat Politèctica de Catalunya).

Norgett, M. J., Robinson, M. T., \& Torrens, I. M. (1975). A proposed method of calculating displacement dose rates. Nuclear Engineering and Design, 33(1), 50-54.

Osetsky, Y. N., Bacon, D. J., Singh, B. N., \& Wirth, B. (2002). Atomistic study of the generation, interaction, accumulation and annihilation of cascade-induced defect clusters. Journal of Nuclear Materials, 307-311(1-4), 852-861.

Schilling, W., \& Ullmaier, H. (2006). Physics of radiation damage in metals. Materials science and technology () Wiley-VCH Verlag GmbH \& Co. KGaA. 